\title{
Multi-objective Optimization of Cognitive Radio Networks
}

\author{
Rodney Martinez Alonso ${ }^{1,2}$, David Plets ${ }^{1}$, Margot Deruyck ${ }^{1}$, Luc Martens ${ }^{1}$, Glauco Guillen \\ Nieto $^{2}$, Wout Joseph ${ }^{1}$ \\ ${ }^{1}$ Information Technology, Ghent University \& IMEC, Technologiepark Zwijnaarde 126, \\ Ghent 9052, Belgium. \\ ${ }^{2}$ R\&D, LACETEL, Rancho Boyeros Ave. 34515, Havana 19200, Cuba. \\ Correspondence should be addressed to Rodney Martinez Alonso; \\ rodney.martinezalonso@ugent.be
}

\section{Keywords}

Cognitive Radio; Spectrum Efficiency; Network Optimization

\begin{abstract}
New generation networks, based on Cognitive Radio technology, allow dynamic allocation of the spectrum, alleviating spectrum scarcity. These networks also have a resilient potential for dynamic operation for energy saving. In this paper, we present a novel wireless network optimization algorithm for cognitive radio networks based on a cloud sharing-decision mechanism. Three Key Performance Indicators (KPIs) were optimized: spectrum usage, power consumption, and exposure. For a realistic suburban scenario in Ghent city, Belgium, we determine the optimal trade-off between the KPIs. Compared to a traditional Cognitive Radio network design, our optimization algorithm for the cloud-based architecture reduced the network power consumption by $27.5 \%$, the average global exposure by $34.3 \%$, and spectrum usage by $34.5 \%$ at the same time. Even for the worst-case optimization (worst achieved result of a single KPI), our solution performs better than the traditional architecture by $4.8 \%$ in terms of network power consumption, $7.3 \%$ in terms of spectrum usage, and $4.3 \%$ in terms of global exposure.
\end{abstract}

\section{Introduction}

The lack of spectrum availability for satisfying the exponential increase in wireless traffic demand has become a major concern in the wireless communication community. Paradoxically, several extensive spectrum usage measurement campaigns have demonstrated that most of the radio frequency spectrum is not in use or is sub-utilized. According to these spectrum surveys performed in cities worldwide, the average use of the sub-3-GHz spectrum regarding both space and time is rarely higher than $20 \%[1,2,3,4]$.

In this context, Cognitive Radio has become a flexible solution to overcome spectrum unavailability by opportunistically exploiting underutilized or unutilized spectrum, particularly in the television broadcasting bands (TV white spaces, TVWS) [5, 6, 7]. Several standardization efforts have been made, e.g., IEEE 802.11 af [8], IEEE 802.22 [9, 10]. Also, for the utilization of TVWS by LTE networks and beyond, the European Telecommunications Standard Institute (ETSI) technical report TR 103 067/2013 [11] analyzed the feasibility of LTE Cognitive Radio Systems operating in TVWS. 
Because of "spectrum scarcity", the bandwidth requirements for next-generation radio and $5 \mathrm{G}$ networks are only fully satisfied at the highest spectrum bands. As a consequence, several applications for 5G networks are limited in rural scenarios, small suburban cities, and in general, in areas with low population density. The feasibility of 5G networks based on Cognitive Radio technology and carrier aggregation, enabling wideband access in rural areas, has also been investigated. Hence, Cognitive Radio is a key enabling technology for new generation radio [12].

A major concern with Cognitive Radio is that interference management has been a challenge for scenarios with dense spectrum occupancy or high user density $[13,14,7]$. The currently implemented standards have some limitations for assessing the trade-off between interference and spectrum usage efficiency. For instance, IEEE 802.11f does not specify any spectrum sensing requirements to be met by user devices and base stations (BSs) for further spectrum allocation [8]. Although geolocation databases allow minimizing interference to the primary licensed services (generally digital television broadcasting), spectrum sensing and spectrum allocation management have to be implemented to avoid interference to or from other Cognitive Radio devices in the same network or other Cognitive Radio networks. These databases are based on measurement campaigns and spectrum usage surveys. Hence, the spectrum usage information is not updated dynamically, reducing the potential spectrum sharing efficiency of cognitive radio networks. IEEE $802.22 \mathrm{~b}$ provides some guidelines and mandatory channel sensing requirements. For instance, it defines the minimum requirements for the scheduling of sensing windows and quiet periods, mandatory users' device reports of detected interference, and maximum interference thresholds for different signal types [10]. However, mandatory sensing techniques are not defined [15].

Several advances on spectrum sensing techniques for avoiding interference have been reported in recent years. In [16], the authors presented a cooperative scheme for spectrum sharing based on the information provided by secondary Wi-Fi nodes. Different improvements for increasing detection efficiency, reducing interference, and exploration time are presented in $[17,18,19]$. A learning algorithm to improve spectrum exploration and to reduce the interference caused by cognitive radio devices is presented in [20]. [21] proposes a learning algorithm to maximize the network throughput by allowing varying sensing time and considering the historical behavior of the user's devices. However, the improvement of the sensing and detection accuracy by itself did not solve the trade-off between allowable interference and spectrum usage efficiency. For this reason, in many cases, regulators ruled the utilization of geolocation databases. These databases contribute to reducing interference but at the cost of a lower spectrum usage efficiency. No major advances have been reported on architecture and dynamic network optimization, being a limitation for a better trade-off between interference management and spectrum usage efficiency.

Few advances have been made on architecture and network medium access and connectivity efficiency to optimize the network's Key Performance Indicators (KPIs). In [22], the authors present a novel cooperative system for the efficient utilization of TVWS based on an Internet of Things (IoT) sensing network. The proposed multilayer architecture improves coexistence issues and the protection of the primary services by combining spectrum sensing and a QoS feedback procedure implemented throughout a control logic in an IoT social platform (where all devices share performance information) [22]. However, this approach requires additional IoT devices for the exchange of information through a third network (IoT platform). Despite 
this, a centralized management approach could be an interesting solution for improving spectrum usage efficiency and coexistence between different services if the same Cognitive Radio platform is used rather than an additional platform.

Besides spectrum usage efficiency, power consumption, and exposure of human beings to radio radiation are important network KPIs. These indicators are closely related to the environmental footprint of Information and Communication Technologies (ICT) [23, 24]. Hence, to achieve environment-friendly wireless networks, it is also required to optimize power consumption and exposure. However, these parameters require the assessment of a trade-off [25, 26, 27]. Also, in [28] authors developed a heuristic method for maximizing the energy efficiency in cognitive sensor networks by optimizing the sensing time and symbol sequence length. Nevertheless, as the spectrum frequency allocation plays a major role in energy efficiency, further research requires a multi-objective assessment of the optimization problem. In this regard, [29] presents a method for the identification of multi-objective optimal settings on a wireless experimentation facility.

The novelty of this paper is the multi-objective optimization of new generation Cognitive Radio networks. Instead of a traditional distributed architecture for spectrum management, we consider a cloud-based architecture. In the traditional cognitive radio architecture, the allocation of spectrum and decisions on the user connections are made by each BS independently. The BSs only have information regarding the spectrum usage and interference from the devices within their reach. Hence, each decision is uncoordinated and independently taken based on limited information on the spectrum and propagation conditions. In the proposed cloud-based architecture the information retrieved by the BSs from each network device is assessed in a centralized Access Controller by a multi-objective optimization algorithm. Based on the global knowledge of the propagation conditions, spectrum usage, and interference perceived by all devices, it is possible to dynamically optimize the network, achieving a higher network efficiency in terms of power consumption, spectrum usage, and global exposure. The dynamic optimization of the network is required for improving its KPIs and reducing harmful interference from/to the primary licensed service. There is no previous research, according to the authors' knowledge, on networking optimization to account for the trade-off among power consumption, spectrum usage efficiency, and exposure for Cognitive Radio in a cloud-based architecture.

The outline of this paper is as follows. In Section 2, we describe the proposed cloud-based architecture for managing Cognitive Radio networks, briefly introduce Pareto optimization, define metrics, details of rationale, describe the multi-objective optimization algorithm, and a description of a realistic scenario and initial wireless network setup considerations. In Section 3, we present the network optimization results based on the proposed architecture and algorithm and benchmark the result against a traditional Cognitive Radio network. Conclusions are presented in Section 4.

\section{Method}

\subsection{Cloud-based architecture for Cognitive Radio network management}

Fig. 1 shows the proposed cloud-based architecture for the Cognitive Radio network that will allow achieving a higher multi-objective optimization level (Fig.1b) compared to the traditional architecture $[8,9,10]$ (Fig.1a). 


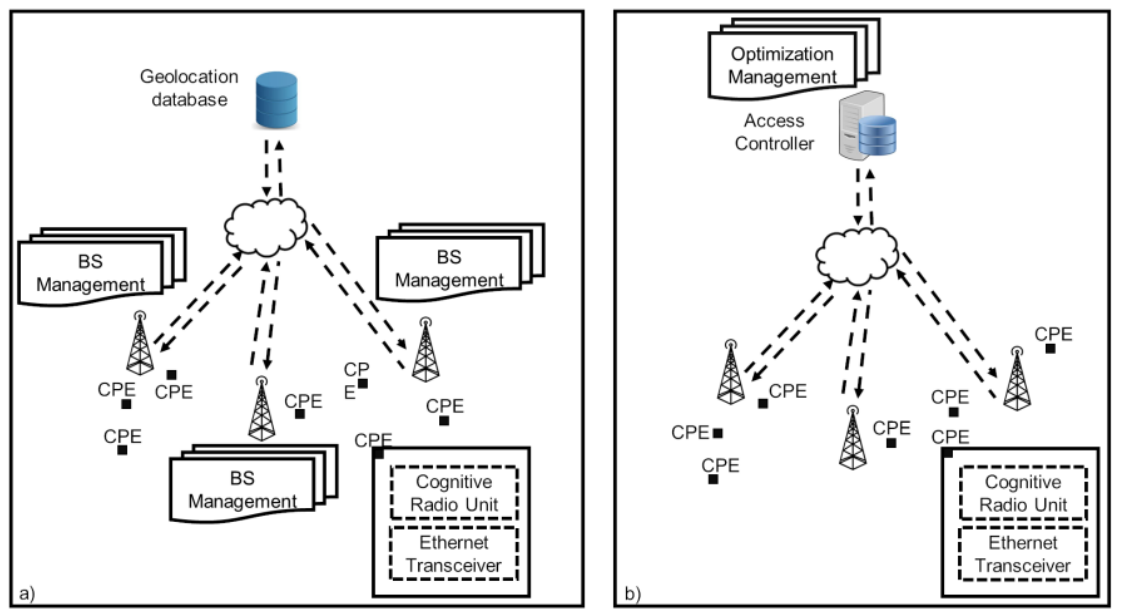

Fig. 1. Cognitive Radio network architecture a) Traditional [9] b) Cloud-based management.

The traditional Cognitive Radio network architecture (in Fig.1a) comprises at the user side the Consumer Premise Equipment (CPE), including an ethernet transceiver and the Cognitive Radio unit. The architecture also includes the BSs, including the BS management or system processing unit for handling the users' data registration, tracking, and application of spectrum management policies defined in the standard. Finally, a connection to ethernet or optical fiber provides access to the internet and (if applicable) to a geolocation database. In this topology (Fig. 1a), each BS oversees users' connections, registration, and tracking and the application of spectrum management policies. BSs take independent decisions about spectrum allocation based on self-sensing and data provided by users in their range. The provision of a geolocation database restricts the degrees of freedom of the BS, forbidding access to certain channels, footprint limitations, and other regulatory policies. These databases are based on static data from spectrum surveys (no real-time updates). The variations on the propagation conditions produce a variation on perceived network interference and spectrum usage efficiency.

Our architecture modification proposal for new generation Cognitive Radio networks consists of moving most management functions (mainly those related to spectrum management) to a central Access Controller (Fig.1b). In this way, it is possible to collect the information sensed by all network devices. By knowing and processing the network performance parameters from the whole network, it is possible to make better decisions (optimization) for serving the users' traffic with higher efficiency regarding the network power consumption, global exposure, and spectrum usage [30]. This information is sent by all BSs to the central Access Controller by the BS data backbone to the cloud. We assume the signalization data related to the Cognitive Radio functionalities is negligible compared to the backbone data capacity. The collected information is the same provided by the geolocation databases to the BSs. Hence, an adaptation of the headers and interfaces defined by the standards is not required.

Although a distributed network topology is generally efficient, if the devices taking decisions are affected by devices outside their control the system becomes unstable. This is similar to the concept of automated vehicular driving in $5 \mathrm{G}$ networks. When the network topology is based on Vehicular-to-Vehicular (V2V) communication, each vehicle only has information about the vehicles in its range, and the system quickly becomes unstable (cars start a crashing chain). This is because each car's control decision is based on the individual knowledge of 
the environment [30]. The same happens in the traditional Cognitive Radio network, where a crash corresponds to interference, and the same happens in any open-loop control system.

\subsection{Pareto Efficiency}

Pareto optimal is a concept of efficiency initially applied in social science and economic problems. The Pareto optimal state is defined as a state where it is not possible to make a single objective (parameter) better without making at least another one worse [31]. In engineering, usually more than one parameter needs to be maximized (multi-objective optimization problems). For a set of choices and a metric to value them, it is possible to find a set that is Pareto efficient. This set is named the Pareto Front [29]. Hence, it is possible to find a set of optimal trade-offs among all parameters depending on the design constraints, scenario, and application.

Wireless networks have several opposing performance indicators, e.g., throughput, energy, latency, electromagnetic radiation, and spectrum usage [29]. The maximization of a certain parameter leads to the minimization of at least one other. This is generally a condition that is not optimal in many wireless applications. By using Pareto optimization, it is possible to evaluate several combinations of performance indicators, each one with a certain weight (Pareto coefficient) in the optimization algorithm (see Section 2.5). In this way, the general Pareto equation $P$ can be defined as a set of $n$ independent metrics $g$ multiplied by a certain weight $w$.

$$
P\left(w_{1} ; w_{2} ; \ldots ; w_{n}\right)=\left\{w_{1} \cdot g_{1} ; w_{2} \cdot g_{2} ; \ldots ; w_{n} \cdot g_{n}\right\}
$$

where for any combination of $w_{1} ; w_{2} ; \ldots ; w_{n}$ the following condition must be satisfied:

$$
\sum_{i=1}^{n} w_{i}=1
$$

We investigate the trade-off among three Key Performance Indicators (KPIs) in a cognitive radio network based on the IEEE 802.22 standard by means of Pareto optimization, being the network power consumption, human exposure, and spectrum usage.

\subsection{Rationale}

In a cognitive network, each device has to sense the spectrum and provide to nearby BSs information related to perceived interference. This information is sent over a wireless link using BPSK modulation in a channel sensed as empty channel to the nearby BSs. The first BS processing the user connection request and allocating the spectrum resources will register the user. In our proposal, this information is assumed to be collected by the BSs, but the allocation of spectrum resources and interference management will be handled by a central access controller. Fig. 2 describes the process of connecting a single user to a Cognitive Radio BS. 


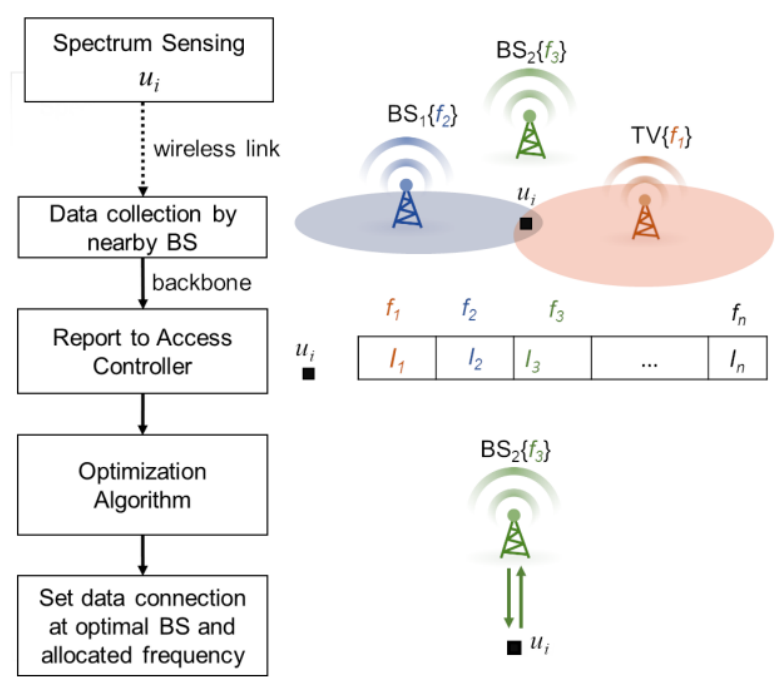

Fig. 2. Flow chart of the steps for connecting a user to a BS in the cloud-based Cognitive Radio architecture.

First, the user devices $u_{i}$ will sense the spectrum and will provide the perceived interference from other BSs, other users, and broadcasting stations to a central access controller (i.e., $I_{l}$, $I_{2}, I_{3}, \ldots, I_{n}$ in Fig. 2). In addition, the user provides information about the radiated signal, geolocation information, and other parameters required by the standard [9] or by regulatory authorities. Notice that the initial link is settled with a nearby BS by using any frequency perceived by $u_{i}$ as free and BPSK modulation for minimum interference [9]. Further, the algorithm must assess the information received from all users and BSs, to compare the perceived interference with the maximum interference signal level allowed (ISL [dBm]) and settle the best connection for $u_{i}$ taking into account the network KPIs. The ISL is the maximum signal level for which the algorithm will allow reusing the same frequencies (i.e., $f_{1}, f_{2}, f_{3}, \ldots, f_{n}$, in Fig. 2) by different BSs. One ISL constraint is defined for sharing frequencies used by television broadcasting (e.g., $T V$ in Fig. 2) and another value for sharing frequencies in use by Cognitive Radio BSs (e.g., $B S_{1}$ and $B S_{2}$ in Fig. 2). Notice that the spectrum allocation is settled for each user. A certain BS can use different frequencies for communicating with different users at the same time, depending on the ISL constraint. For instance, if the interference is higher than the maximum allowable $B S_{2}$ and $u_{i}$ (see Fig. 2) must communicate in a frequency not in use by $B S_{1}$ or $T V$ (i.e., $f_{3}$ ). Otherwise, it can reuse the same frequency (i.e., $f_{1}$ or $f_{2}$ ).

For the greenfield network planning, a reduction in the number of BSs has a major impact on the minimization of the network power consumption and cost. This is because the Cognitive Radio BSs have an idle or fixed power consumption that is not directly related to the radiated power or with the traffic load. For instance, for peak traffic and maximum radiated signal level, the Cognitive Radio BS power consumption is $64 \mathrm{~W}$ versus $38 \mathrm{~W}$ without data traffic (idle power consumption is approximately 59\%) [32]. Additionally, the architecture with a centralized access control allows dynamically switching a group of BSs from active to sleep mode. The Cognitive Radio BS has a power consumption of only $9 \mathrm{~W}$ in sleep mode. However, by reducing the number of BSs, the radiated power per BS increases to cover the most distant users. As a consequence, the exposure in that cell increases [25]. In this way, the optimization algorithm should follow different optimization strategies, depending on the parameters to be optimized. More details of the optimization algorithm are provided in Section 2.5). 


\subsection{Metrics}

First, we defined the following metrics for accounting in the algorithm for the KPIs intended to be optimized, i.e., network power consumption, network global exposure, and spectrum usage.

\subsubsection{Power Consumption}

The power consumption for a network configuration is accounted for following the power consumption model presented in [32] for a Cognitive Radio BS. Here, when accounting for the network power consumption, we consider that the centralized access controller (see Fig. 1) can switch the BSs that are not in use at a certain instant of time to sleep mode. In sleep mode, the Cognitive Radio BS has a power consumption as low as $9 \mathrm{~W}$, including the radio unit, optical backhaul, and electrical transceiver. Notice that without centralized control, not all power-consuming components can be switched to sleep mode.

\subsubsection{Network Global Exposure}

We consider the Electric field (E-field) in each pixel of the map as the metric for characterizing the exposure caused by all radiating sources in the network (i.e., network global exposure). The E-field is commonly used for exposure characterization, as it is independent of the exposed person. As such, the E-field unambiguously allows assessing the exposure due to a given wireless network [33]. Hence, the global exposure $E_{G}$ is defined as a weighted average of the mean electric field $E_{50}$ and the 95-percentile of the field strength $E_{95}$ over the covered area [25], in order to optimize median and maximal exposure values. As in [23], we consider an equally weighted $E_{50}$ and $E_{95}$. Hence, the $E_{G}$ can be described by the following equation:

$$
E_{G}=\frac{E_{50}+E_{95}}{2}
$$

For calculating the electric field strength over the covered area, a grid of "test points" separated $50 \mathrm{~m}$ from each other is generated covering the entire map. At each grid point, the contribution of each transmitter $T x_{j}$ to the electric field strength is calculated. The electric field $E_{T x}[\mathrm{~V} / \mathrm{m}]$ due to transmitter $T x_{j}$ can be calculated based on $T x_{j}$ 's Equivalent Isotropically Radiated Power EIRP [dBm], frequency $f[\mathrm{MHz}]$, and the path loss $P L[\mathrm{~dB}]$ from the $T x_{j}$ to the grid point (x,y) [25], as described by Equation 4.

$$
E_{T x_{j}}(x, y)=10^{\left(\frac{\operatorname{EIRP}_{T x_{j}}-43.15+20 \cdot \log _{10}(f)-P L_{T x_{j}}(x, y)}{20}\right)}
$$

The contribution of all transmitters to the electric field strength at each grid point $(x, y)$ is calculated by accounting for the root sum of the squares of the electric field strengths due to each $T x_{j}[25]$.

$$
E_{\text {total }}(x, y)=\sqrt{\sum_{j=1}^{n}\left[E_{T x_{j}}(x, y)\right]^{2}}
$$


where $n$ is the total number of transmitters. The field vectors caused by each source are thus assumed to have no phase correlation [25].

\subsubsection{Spectrum usage}

We define the spectrum usage $S_{U}$ as the number of channels that are required by the network for settling all simultaneous connections in the deployment scenario.

$$
S_{U}\left(u_{i}, B S_{j}\right)=\sum_{c h=1}^{\operatorname{Smax}} k_{c h}\left(u_{i}, B S_{j}\right)
$$

where $k_{c h}$ is 1 if the channel $c h$ has been assigned to a communication link between at least one user $u_{i}$ and a $B S_{j}$, else $k_{c h}=0 . S_{\max }$ is the maximum number of channels allowed to be used according to the regulatory domain. The spectrum optimization is performed by reusing channels in the communication between each user and the BS. The metric $S_{U}$ is a measure of the spectrum occupation at the BS and user locations. Each user device dynamically accesses the spectrum at the most suitable frequency. A single BS can communicate with its connected users by means of different frequencies. The frequency channels are reused when the interference constraints (ISL) defined in the standard [9] are accomplished.

For comparing the spectrum usage efficiency (SUE $\in[0: 1])$ between the proposed cloudbased architecture and the traditional distributed architecture for cognitive radio, we quantify the spectrum usage efficiency as the ratio between the number of channels that are really in use by the radiocommunication systems $\left[S_{U}\right]$ and the maximum allocated spectrum by the regulators (in terms of channels) in the UHF bands $\left[S_{\max }\right]$. The following equation allows quantifying the spectrum usage efficiency.

$$
S U E=1-\frac{S_{U}}{S_{\max }}
$$

\subsubsection{White Space Availability}

For a fair comparison among different solutions, we also define the white space availability in the whole area.

$$
W a(x, y)=S_{\max }-S_{U T x}(x, y)
$$

The white space availability $W a$ represents the number of channels available at each grid point $(\mathrm{x}, \mathrm{y})$ after the access controller assigned the spectrum for all links among the BSs and the users and also accounting for television stations. For calculating this value the whole area is divided into grid points with coordinates $x, y$ (considering a resolution of $50 \mathrm{~m}$ ). $W a$ is the difference between the total number of channels $S_{\max }$ and the number of channels in use by the Cognitive Radio network devices and the channels in use by the television broadcasting service $S_{U T x}$ at each grid point. Notice that $S_{U}$ accounts only for the Cognitive Radio network spectrum usage, and $S_{U T x}$ also includes the television broadcasting spectrum usage. Hence, $W a$ represents the remaining channel availability in the area. The mean white space availability can be calculated accounting for $W a$ in all grid points. 


\subsection{Multi-objective Optimization Algorithm}

Algorithm 1 describes the network optimization algorithm that will be used for minimizing the network power consumption, spectrum usage, and exposure (i.e., goal KPIs). The algorithm is heuristic and capacity-based [23]. Hence, we cannot guarantee an absolute optimal network solution but a solution that is good enough for solving the optimization problem. The solution convergence is defined by a maximum $2 \%$ standard deviation of the progressive average for each optimized network KPIs (spectrum, power consumption, and exposure). The lower the number of users per square kilometre, the more diverse the possible distributions of those users, and the higher the number of possible solutions. Notice that interference and spectrum management is not included in the base algorithm described in $[23,24]$. Hence, modifications were performed for the multi-objective optimization goals for this work.

The base algorithm in [23, 24] was divided into two phases. One phase comprises the greenfield network planning (i.e., designing the network from zero infrastructure based on the capacity and coverage requirements), and a second phase includes the dynamic optimization of the considered KPIs. The main contributions to the algorithm design are the inclusion of dynamic spectrum allocation for the BSs based on interference constraints and an extension for operating in a cloud-based architecture assessing the data from all network devices. Here, the spectrum usage efficiency is also optimized as spectrum usage efficiency is a KPI in the algorithm fitness function. Also, the automation of the selection of BSs locations based on a histogram allows a faster and more efficient design of the initial network planning.

\subsubsection{Phase 1: Greenfield network planning}

For the greenfield planning and BS locations selection, the network traffic and coverage are modeled by Phase 1 of the optimization algorithm. First, the users and traffic requirements are input parameters. The algorithm first generates a uniform and pseudo-random distribution of the users in the area and assign a traffic load per user (see Phase 1 line 2 in Algorithm 1, the initial values for the input parameters are described in Section 2.6). Notice that the whole algorithm is repeated for a maximum number of simulations (Max_Sim). For the greenfield planning, an initial set of BSs (see initial values of input parameters in Section 2.6) is optimized to find the minimum required number of BSs $\left(N_{B S}\right)$ [32] and the best BS locations (in terms of average path loss to users). A histogram with the number of connections settled by each BS during a group of simulations (Max_Sim) is performed, and the optimal BS locations are chosen based on the probability of having the lowest path loss to users (Phase 1 line 5 to 13, in Algorithm 1). The number of simulations Max_Sim is empirically chosen to guarantee that the progressive average of the KPIs has a standard deviation lower than $2 \%$. The output of the first phase is a new set with a number $N_{B S}$ of optimal BS locations (see Phase 1, line 15 in Algorithm 1). 

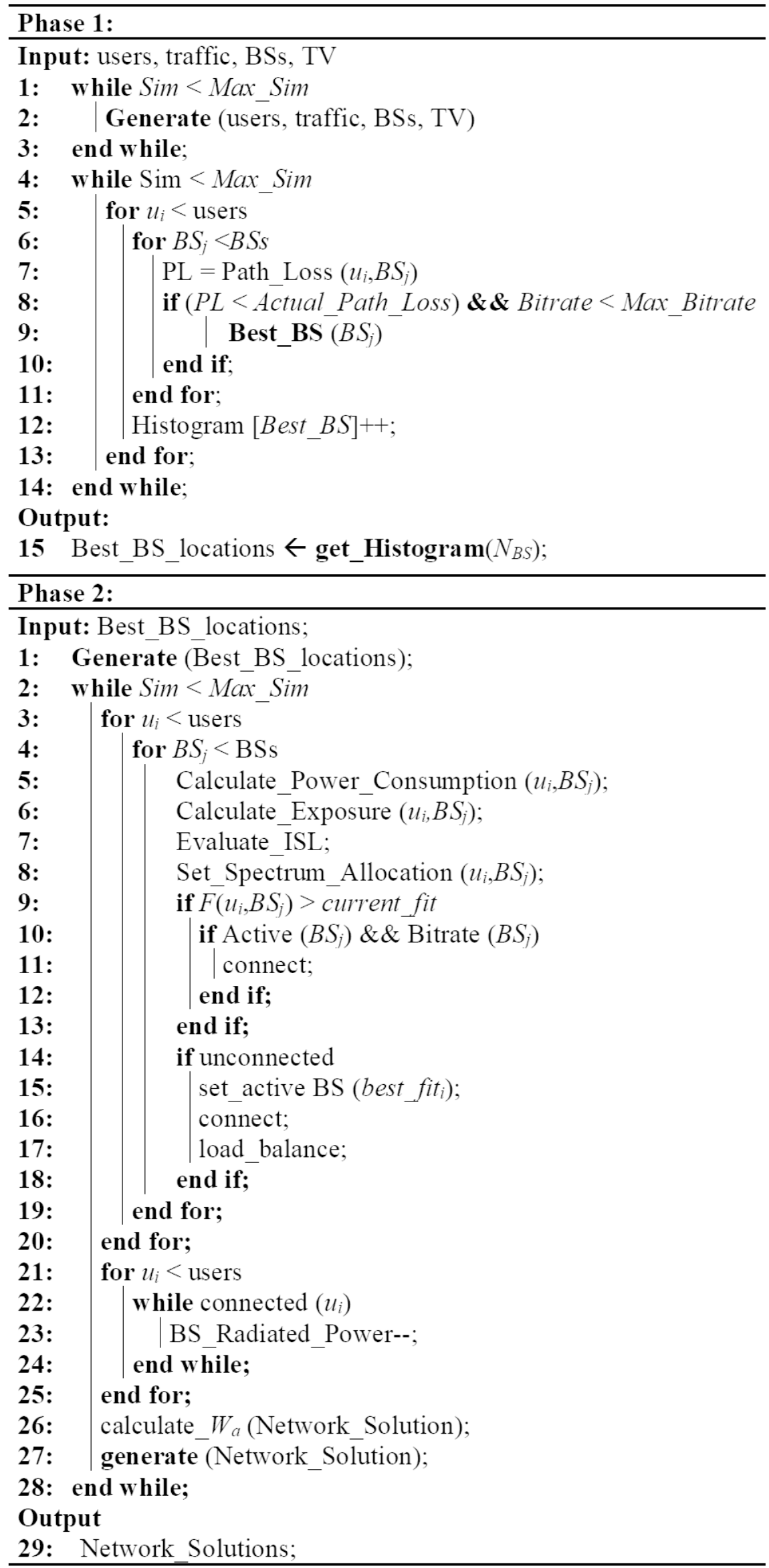

Algorithm 1. Multi-objective optimization algorithm. 


\subsubsection{Phase 2: Dynamic network optimization}

In a second phase the algorithm dynamically optimizes the network KPIs (i.e., spectrum usage, power consumption, and exposure). The second phase receives as input the set of BSs (Best_BS_locations). The users and traffic densities are the same as Phase 1. For each combination of Pareto coefficients (coefficients defining how each KPI is weighted when optimizing the network), a number of Max_Sim simulations with different random distributions of users is performed. For each Pareto coefficient set and simulation (user distributions), a network solution is retrieved with the achieved value for the evaluated KPIs. Each combination of KPIs represents a sample in the whole Pareto population. For the Pareto optimization, several combinations of weights must be evaluated, considering a trade-off between accuracy and computation time. We consider a resolution of 0.25 for the Pareto coefficients.

For the total number of simulations Max_Sim and for each user $\left(u_{i}\right)$, the algorithm calculates a fitness value for each possible connection $B S_{j}$. For each possible connection, the algorithm calculates the Power Consumption, Exposure, and after evaluating a certain Interference Signal Level constraint (ISL), it finds the best spectrum allocation for each link to be settled (Phase 2 lines 5 to 8 in Algorithm 1).

For the network modeling and optimization, the interference levels are calculated based on the radiated power and path loss calculations. We start by evaluating (and further assigning) the lowest available frequency (for a better signal propagation). First, the algorithm will verify the interference level at the user and BS sites generated by all users, all BSs, and television broadcasting towers in the surroundings. This is possible because we consider a centralized access architecture retrieving information from all devices. Hence, the decision on frequency assignment is based on the information provided by all BSs from all users. Notice that in the traditional Cognitive Radio network architecture, the BSs are in charge of the frequency allocations taking into consideration static information from a geolocation database and the interference level information provided only by users in their range.

Fitness Function: The fitness function fit (Phase 2 line 9 in Algorithm 1) accounts for the network power consumption $P_{C}[\mathrm{~W}]$, global exposure $E_{G}[\mathrm{~V} / \mathrm{m}]$ [23], and spectrum usage $S_{U}$ if the user $u_{i}$ is connected to a certain $B S_{j}$ (see metrics defined in Section 2.4).

$$
f i t\left(u_{i} ; B S_{j}\right)=w_{1} \cdot\left(1-\frac{P_{C}}{P_{\max }}\right)+w_{2} \cdot\left(1-\frac{E_{G}}{E_{\max }}\right)+w_{3} \cdot\left(1-\frac{S_{U}}{S_{\max }}\right)
$$

where $P_{\max }$ is the maximum power consumed by the network (i.e., all BSs active with a maximum radiated power); $E_{\max }$ is the maximal exposure over the considered area for the same network conditions. In this way, all performance indicators are normalized, and no parameter is overrated. Hence, for the worst-case $P_{C}=P_{\max }, E_{G}=E_{\max }$, and $S_{U}=S_{\max }$, the fitness function equals 0 . The weight factors are defined as $w_{1}, w_{2}$, and $w_{3}$. These weight factors corresponding to the Pareto coefficients and range from 0 to 1 .

Each user is connected to the BS with the highest fitness value (lowest power consumption, spectrum usage, and exposure), if this BS is already active and still can support the user's demanded bitrate (Phase 2 lines 9 to 13 in Algorithm 1). If no active BS can support the 
user's demanded bitrate, a new BS with the best fitness value is switched active. For balancing the network load, already connected users can be switched to this new active BS if their fitness value to this BS is higher than before (Phase 2 lines 14 to 18 in Algorithm 1). Once all users have been evaluated, the first network solution is optimized by decreasing the BS radiated power (Phase 2, lines 21 to 25 in Algorithm 1). The stop condition is reached when the path loss experienced by a user is higher than the maximum allowable path loss [23]. The decrease of the radiated power will decrease the power consumption, exposure and will allow a better re-usage of the spectrum.

The algorithm will calculate the network solutions white space availability ( $W a$ ) for generating a map with the spectrum availability (Phase 2, line 26 in Algorithm 1). Finally, the algorithm will generate the network solutions (Phase 2, line 27 in Algorithm 1). For each network solution, the KPIs are calculated for the whole network. After all the network solutions are retrieved, the whole Pareto population is mapped. Each pixel in the map represents the KPIs for a certain Pareto weight combination and a unique random distribution of the users in the area (Pareto population). In this way, it is possible to assess the network performance for each KPI and find the best trade-off between these KPIs (Pareto front). The Pareto frontier will be defined by a surface where every single parameter leads to the KPIs minima.

Because the Pareto optimization in the designed heuristic algorithm takes a finite number of weights, the missing pixels in the three-dimensional optimization map are interpolated using Delaunay triangulation.

\subsection{Evaluation scenario and initial setup}

To validate the proposed architecture and optimization algorithm, we modeled and optimized a Cognitive Radio network in a real suburban wireless scenario. We consider the city of Ghent, Belgium $\left(68 \mathrm{~km}^{2}\right)$ for the greenfield planning and later dynamic optimization of the network. Fig. 3b shows a map of Ghent City and the BS possible locations denoted with dots. Fig. 3a shows a map of the Region of Flanders, Belgium covering an area of approximately $13,522 \mathrm{~km}^{2}$ with the location of the television broadcast transmitters. 


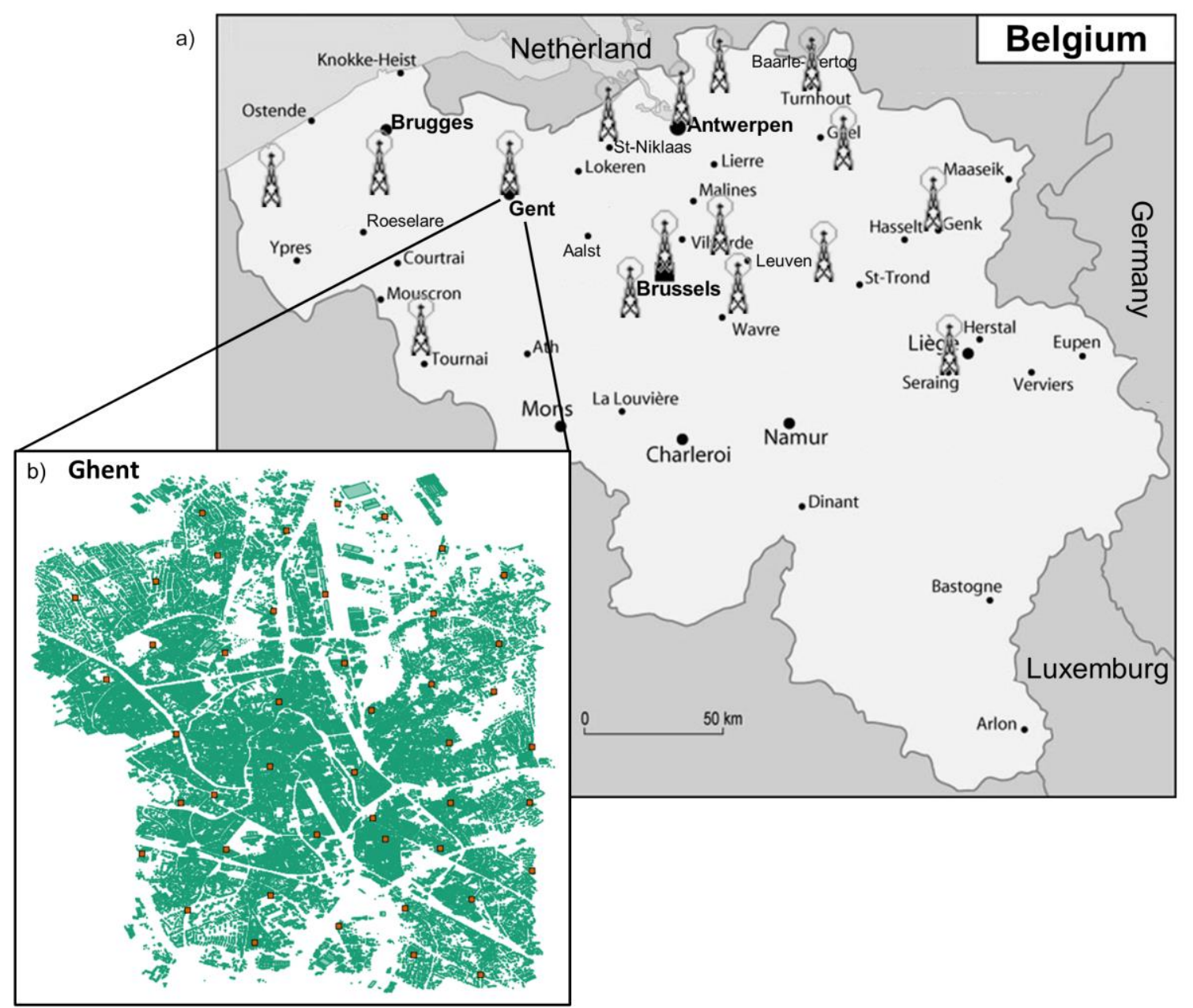

Fig. 3. a) Broadcast transmitters in the region of Flanders, Belgium, and b) area to be covered in Ghent, City, 45 possible BS locations identified with dots.

A traditional Cognitive Radio network design is also modeled for a comparison reference. For this network, it is not possible to implement the sleep mode. This is because in sleep mode the spectrum management and user tracking in the BS are switched off. Without a centralized access controller capable of assuming spectrum management and tracking functions the Cognitive Radio BS can only be switched to idle. In idle mode, the BSs implement most sensing functions, signaling, and tracking, but no user data payload traffic is handled.

A set of 45 possible locations is considered for the Cognitive Radio BSs (represented by squares in Fig. 3b). After the first optimization step, the algorithm retrieves a histogram of the optimal BSs locations and determines the minimum number required to satisfy the intended coverage $\left(N_{B S}\right.$, see Section 2.5.1). The network is designed to guarantee $95 \%$ coverage at the cell-edge during $99 \%$ of the time. We consider the link budget presented in [32] for the IEEE 802.22 standard.

We consider 224 simultaneous user connections at the peak traffic time and a bit rate of $1 \mathrm{Mbps}$ per user [32]. Three scenarios are modeled, and the networks are dynamically optimized for three densities of BS infrastructure availability, i.e., the minimum number of BSs that satisfies the coverage constraint $\left(N_{B S}\right), 25 \%$, and $50 \%$ higher BS density. In all cases, for the green field network planning the BSs are chosen according to the user's connections histogram. 
The algorithm will realize two network solutions for each BS infrastructure availability considering an interference threshold of $-116 \mathrm{dBm}$ and $-93 \mathrm{dBm}$ for the Cognitive Radio sensed signals. The threshold of $-116 \mathrm{dBm}$ is considered if channel occupancy is based on sensing the IEEE 802.22.1 Beacon and sensing mode 0. The threshold of $-93 \mathrm{dBm}$ is defined in the standard for sensing mode zero and signal type IEEE 802.22 WRAN [9]. There is no recommended interference threshold value for DVB-T/T2 digital television signals in the IEEE 802.22 standard $[9,10]$. For evaluating the reusability of frequencies in use by nearby television broadcasting stations, an interference constraint of $-95 \mathrm{dBm}$ is considered. This value is based on the recommended protection contour of the broadcast transmitter [3]. The chosen interference constraint guarantees the minimum carrier-to-interference-ratio recommended in [14] for the protection of the primary service from harmful interference.

All the broadcast transmitters around Ghent (Fig. 3a) are included in the model to account for the interference levels. For the path loss calculations for TV Towers in Flanders, we consider their actual transmitter configurations $[34,35]$ and the ITU path loss model ITU-R P.1546$5 / 2013$ [36]. For the path loss calculations in Ghent, we consider an experimental one-slope path loss model based on an extensive measurement campaign in the UHF band as described in [37]. This model has higher precision for Ghent city than the ITU-R P.1546-5/2013 model.

\section{Results and Discussion}

\subsection{Greenfield network planning: infrastructure density}

Figure 4 shows a histogram of all users' connections to the BSs after 40 simulations considering different random distributions of the users in the area.

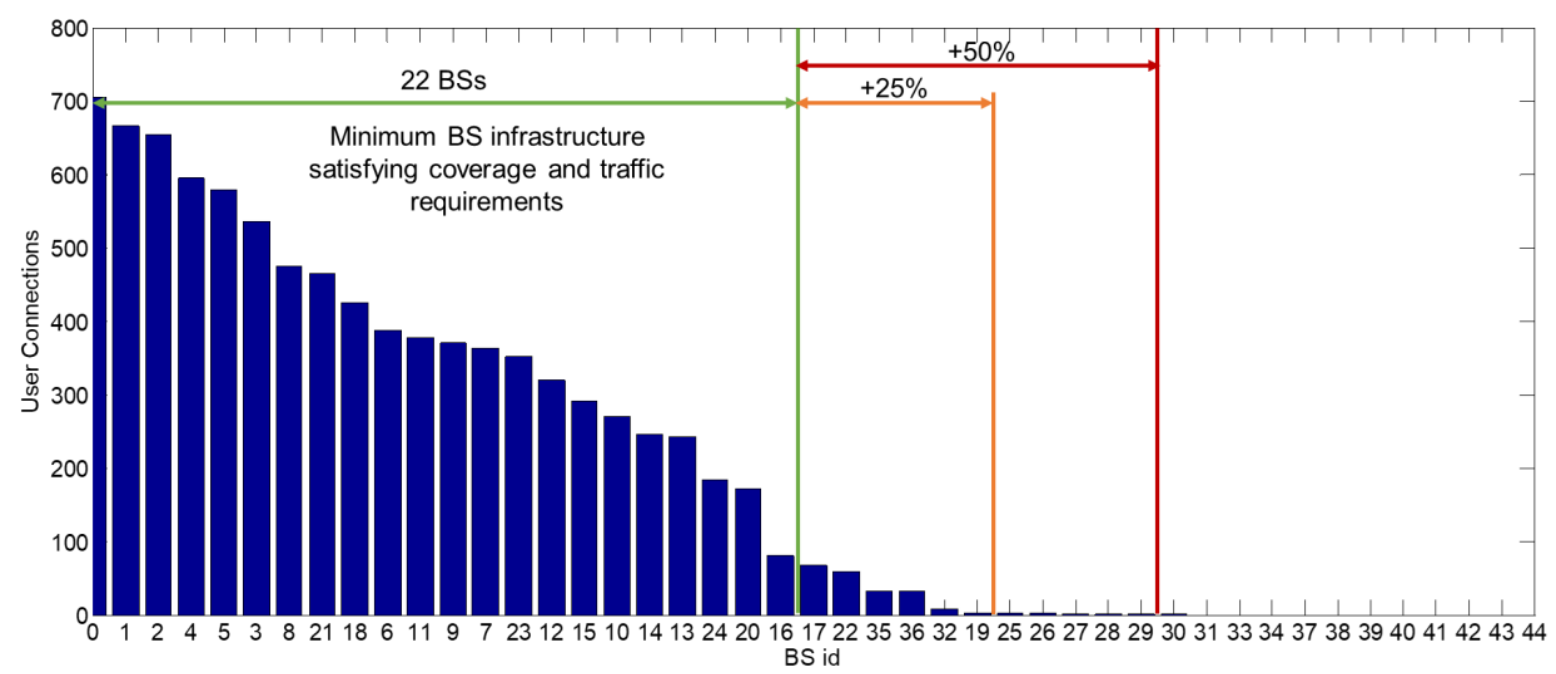

Fig. 4 Network connections histogram

The minimum number of BSs for satisfying the traffic and coverage (spatial and temporal) requirements of the Cognitive Radio network is $N_{B S}=22$. The BS selection is not defined by the starting selection of the algorithm but the relative locations of the BSs and the environmental propagation conditions. The second optimization step, i.e., dynamic network optimization, is realized for the chosen 22 BSs based on the computed histogram (in Fig. 4). The dynamic network optimization from Phase 2 (in Section 2.5.2) is also realized for a $25 \%$ (28 BSs) and 50\% (33 BSs) higher BS infrastructure (BS selection based on the histogram). 


\subsection{Pareto Optimization}

Fig. 5 shows the generated KPI optimization results for the minimum number of BSs to guarantee the coverage requirements $\left(N_{B S}=22\right)$ and considering an Interference Signal Level threshold ISL $=-93 \mathrm{dBm}$.

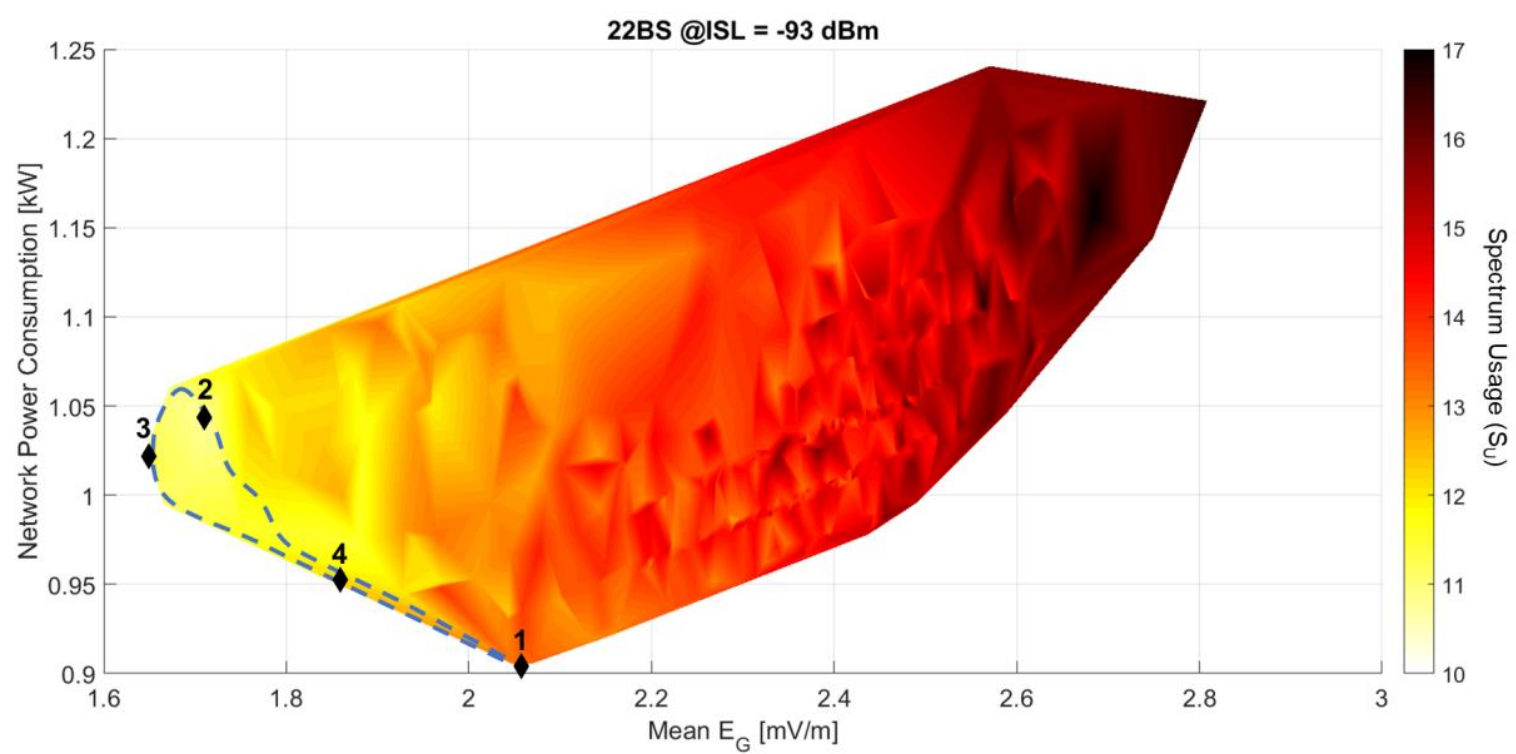

Fig. 5 Dynamic network optimization results for 22 BS locations (minimum to guarantee coverage and traffic requirements), @ ISL = $-93 \mathrm{dBm}$ (2D projection view). The dashed line represents (part) of the Pareto front. Marker 1 denotes the best power consumption results, marker 2 best spectrum usage result, marker 3 best exposure result, and marker 4 optimal trade-off between the KPIs

The best performance in terms of spectrum and global exposure is denoted by markers 2 and 3 , respectively. The KPI results for these Pareto points correspond to the weights (Pareto coefficients), maximizing either the optimization of spectrum usage or network global exposure. The difference between all the KPIs for these two markers is at most 5.3\%. This is because a major impact in both spectrum usage and exposure is achieved for a larger density of active BSs with a low radiated power. However, the power consumption for these network solutions is approximately $15 \%$ higher than the best solution in terms of power consumption (marker 1 in Fig. 5). This difference is caused by the strategies followed by the algorithm for optimizing different KPIs. When the Pareto coefficients for exposure or spectrum optimization are higher, the network solution will lead to an increase in the number of active BSs (serving user traffic) with a lower radiated power and a better load balance. For improving spectrum reusability, a lower radiated signal level will lead to lower spectrum usage but with a more balanced rate than in the case of the network exposure optimization. This is because the ISL constraint has an additional impact on the connection decisions and spectrum allocation. Conversely, when the weight for the power consumption is higher in the fitness function, the algorithm will try to switch to sleep mode BSs that are not required at a certain instant of time for handling the user traffic demand or coverage. This network configuration will lead to lower power consumption at the cost of a lower load balance.

In general, with the increase in the number of active BSs, spectrum and network exposure improve for different points in the Pareto front (surface inside the dashed line), but with the drawback of higher power consumption. This is because the sleep mode of the BS consumes 
only $9 \mathrm{~W}$ (14\% of the maximum power consumption). Hence, a higher optimization in terms of power consumption is achieved when BSs are switched to sleep mode. The best trade-off between all the KPIs (best balance between the optimization strategies) is denoted by marker 4. The drawback of this balanced network solution is an increase between $5 \%$ and $15 \%$ for each KPI, compared to the maximum optimization achievable for a single KPI (markers 1 to $3)$.

\subsection{Impact of the infrastructure density on the network dynamic optimization}

Fig. 6 shows the dynamic optimization results when a) 28 BSs are available (an increase of BS locations by 25\%) b) $33 \mathrm{BSs}$ (an increase of BS locations by 50\%) with an Interference Signal Level constraint ISL $=-93 \mathrm{dBm}$.
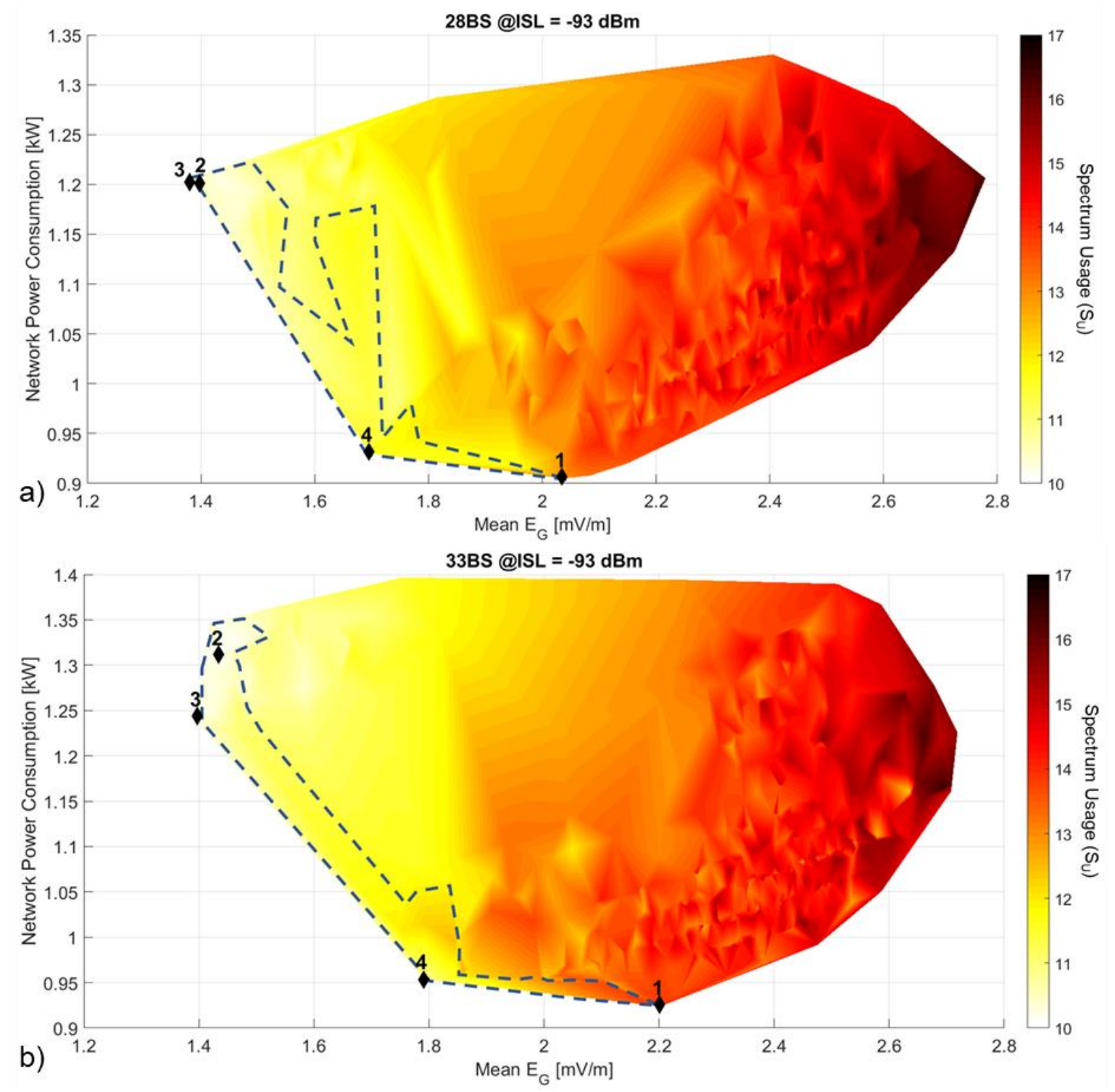

Fig. 6 Optimization results for a) $28 \mathrm{BS}$ and b) $33 \mathrm{BS}$ locations @ ISL = -93 dBm (2D projection view). The dashed line represents (part) of the Pareto front. Marker 1 denotes the best power consumption results, marker 2 best spectrum usage result, marker 3 best exposure result, and marker 4 optimal trade-off between the KPIs.

By increasing the infrastructure availability (more BSs to be switched from sleep mode to 
active if needed) by $25 \%$, the spectrum usage is slightly reduced by $3.7 \%$ (see marker 2 in Fig. 5 vs Fig. 6a), and the global exposure reduces by $16.3 \%$ (see marker 3 in Fig. 5 vs Fig. 6a). An increase of $50 \%$ of BS density (Fig. 6b) reduces the spectrum usage by $5.6 \%$ (see marker 2) and exposure by $15.8 \%$ (see marker 3) compared with 22 BS density (Fig. 5). Notice that here the exposure is similar for 28 BSs and 33 BSs. In this case, the reduction of radiated power per BS does not compensate for the increase of radiating sources (more BSs activated by the algorithm for serving users' traffic). The improvements in spectrum usage and exposure have a drawback on the network power consumption. The power consumption increases by $13.3 \%$ to $15 \%$ for a $25 \%$ higher BS density (Fig. 6a marker 2 and 3) and $17.7 \%$ to $20.6 \%$ for a $50 \%$ higher BS density (Fig. 6b). This is a direct consequence of a higher density of active BSs for the network solutions in Fig. 6a and Fig. 6b.

For the best network solution in terms of power consumption (when the algorithm assigns a higher weight to the power consumption in the fitness function), there is no significant variation on this KPI as the density of available BS locations increases. The network power consumption varies from $0.91 \mathrm{~kW}$ to $0.92 \mathrm{~kW}$ (the difference is lower than the standard deviation). This is because the algorithm switches most of the BSs to sleep mode and increases the radiated power to reach the farthest users. The lower density of active BS (serving user traffic) leads to lower power consumption, even when the radiated power increases. As the density of active BSs decreases, the power consumption is reduced between $30 \%$ and $35 \%$ compared with the maximum network power consumption, but the spectrum increases around $20 \%$ and the exposure between $20 \%$ and $37 \%$ (see marker 1 in Fig. 6 compared to Fig. 5).

The most balanced trade-off between the KPIs is denoted by marker 4 (the weight combination in the algorithm fitness function causes a better balance of the KPIs). Increasing the number of BS locations do not allow a significant improvement of the mean point in the Pareto frontier (marker 4). Notice that for a $25 \%$ higher BS density (see Fig. 6a marker 4), the mean spectrum usage only improves by $3.3 \%$ and the global exposure by $8.6 \%$ while the network power consumption remains almost equal (the difference is lower than the standard deviation). For a 50\% higher BS density (see Fig. 6b marker 4), the mean spectrum usage improves by $10 \%$, but the global exposure only improves by $3.7 \%$ with almost the same network power consumption.

\subsection{Cloud-based vs traditional network architecture}

Fig. 7 shows the difference (in percentages) for each Pareto point in the cloud-based architecture compared to the traditional distributed architecture for $22 \mathrm{BSs}$. 


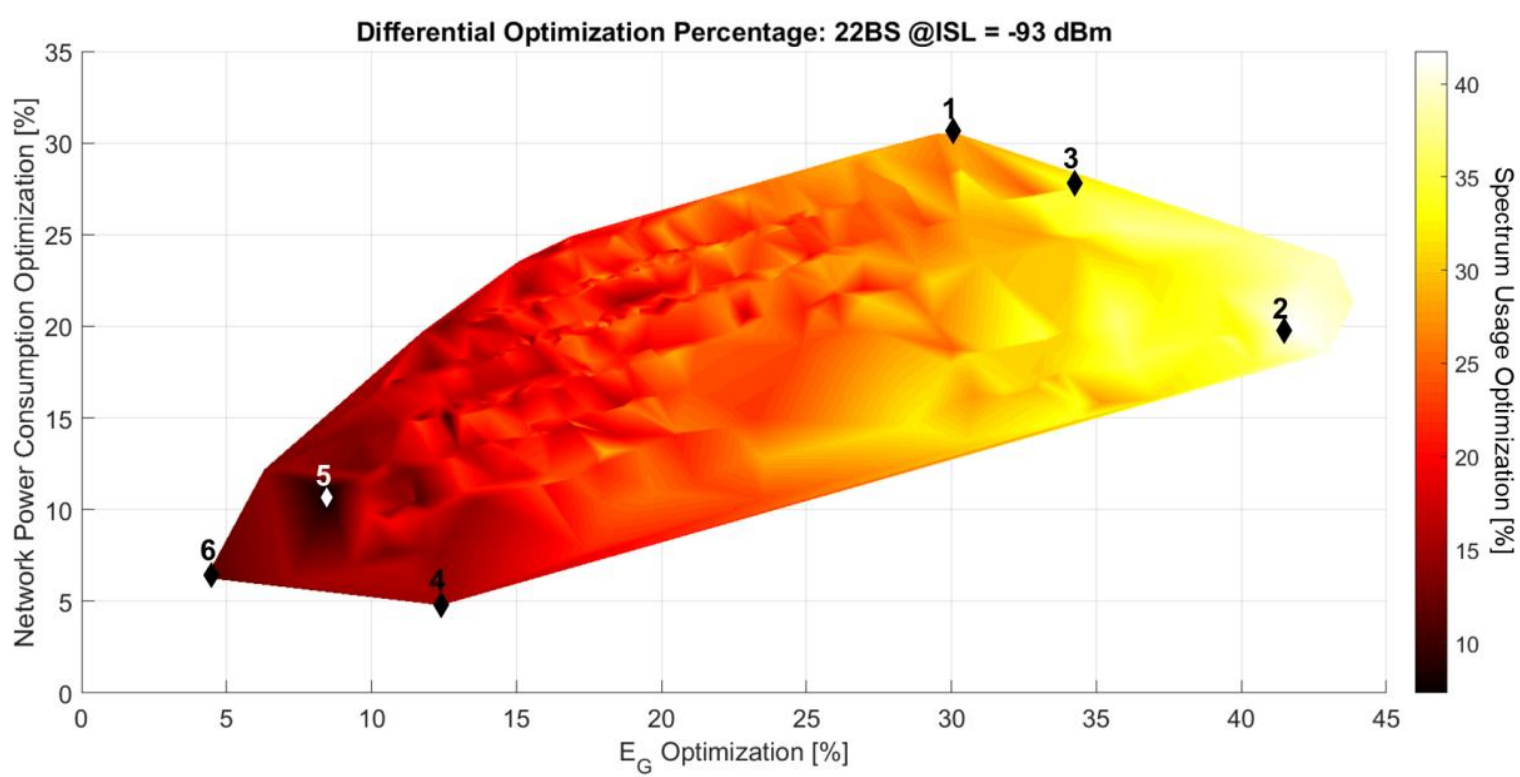

Fig. 7 Differential optimization comparison between the traditional Cognitive Radio network and the results for the cloud-based Cognitive Radio architecture for 22 BS @ ISL $=-93 \mathrm{dBm}$ (2D projection view). Marker 1 denotes the best power consumption result, marker 2 the best spectrum usage result, marker 3 the best exposure result, marker 4 the worst-case power consumption result, marker 5 the worst-case spectrum usage result, and marker 6 the worst-case exposure result.

The network modeling for the traditional Cognitive Radio network and $22 \mathrm{BSs}$, yielded a power consumption of $1.3 \mathrm{~kW}$, a network global exposure of $2.9 \mathrm{mV} / \mathrm{m}$ and mean spectrum usage of 18.4 channels, equivalent to a spectrum usage efficiency of 0.53 . In Fig. 7 we represent the differential values (in percentage) compared to each Pareto point in the optimized cloud-based Cognitive Radio network. For maximum network power consumption savings (power consumption weighted higher in the algorithm fitness function), the cloudbased architecture reduces power consumption by $30.6 \%$ (see marker 1 in Fig. 7). For this network solution the spectrum usage is $28.1 \%$ lower, and the mean global exposure is $30.1 \%$ lower, compared to the traditional Cognitive Radio network architecture. The best trade-off among spectrum usage and mean global exposure (see marker 2 Fig. 7) achieves a higher performance by $41.6 \%$ (for both KPIs) and lower power consumption by $19.9 \%$. The most balanced network solution among all KPIs (marker 3 in Fig. 7) achieves a lower spectrum usage by $34.5 \%$, lower global exposure by $34.3 \%$, and lower network power consumption by $27.5 \%$. In addition, notice that for the worst-case network solution (weight combinations that lead to significant degradation of at least one KPI), the proposed architecture performs better than the traditional Cognitive Radio network at least by $4.8 \%$ in terms of network power consumption (marker 4 in Fig. 7), 7.3\% in terms of spectrum usage (marker 5 in Fig. 7), and $4.3 \%$ in terms of global exposure (marker 6 in Fig. 7). These results are due to the fact that a better connection decision is made by a centralized access controller when assessing the data collected by all devices, rather than an independent decision by each BS based only on information from devices in their service area.

\subsection{Effect of ISL constraint}

Fig. 8 shows the Pareto multi-objective optimization results for $22 \mathrm{BS}$ (minimum to guarantee coverage requirements) with an interference signal level constraint of $-116 \mathrm{dBm}$. 


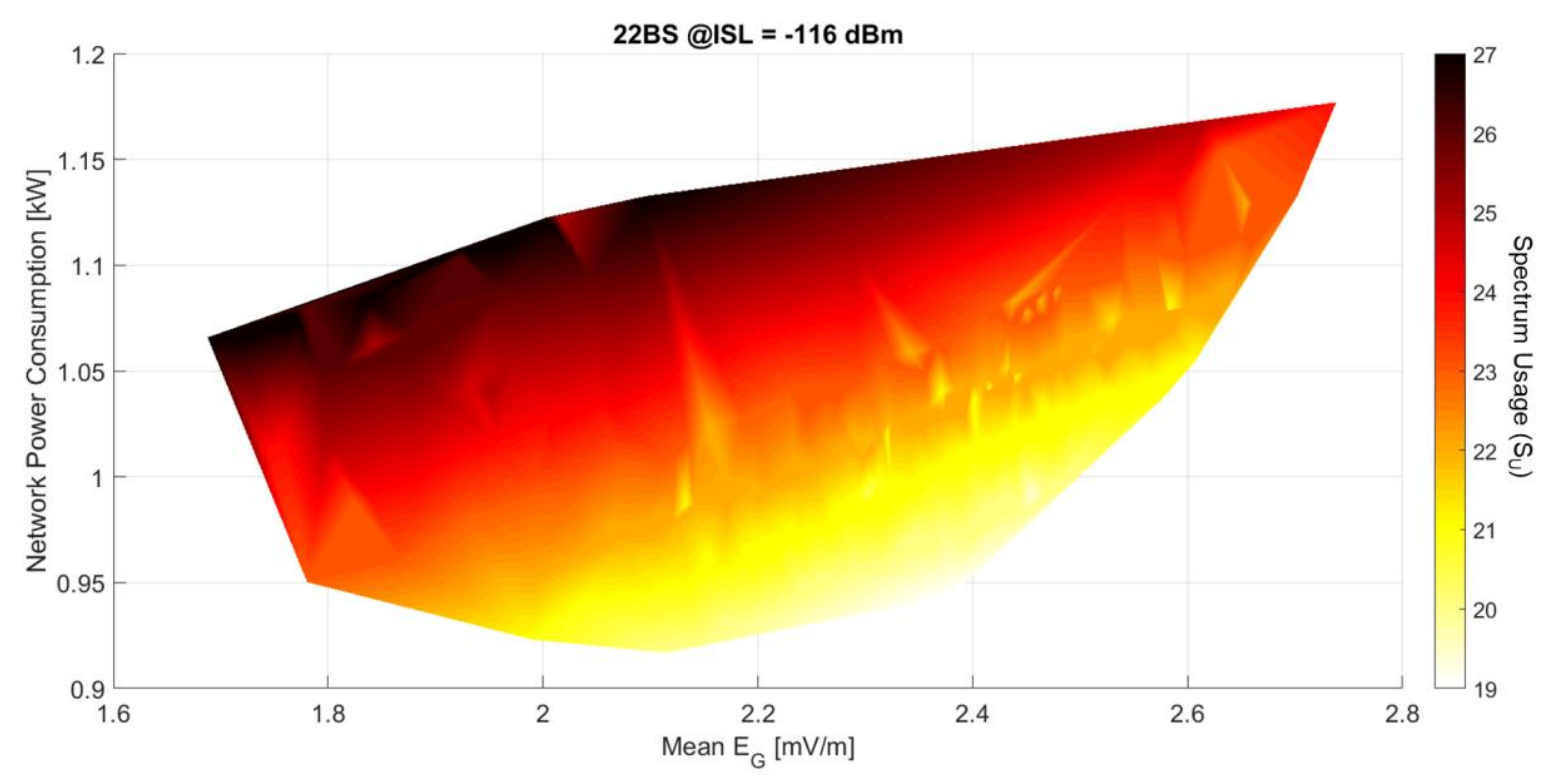

Fig. 8 Pareto optimization results for 22 BSs @ ISL =-116 dBm

The optimization results achieved for the network power consumption and global network exposure are similar in comparison to the results for an interference constraint of $-93 \mathrm{dBm}$ (see results for ISL $=-93 \mathrm{dBm}$ in Fig. 5). However, the spectrum usage is considerably higher. For the best network solution in terms of spectrum usage the $S_{U}$ is equal to 19.0 (equivalent to spectrum usage efficiency of 0.51 ). This value is $43.7 \%$ worse than the best spectrum usage results for $22 \mathrm{BSs}$ and ISL $=-93 \mathrm{dBm}$, and $10 \%$ worse compared to the worst case in terms of spectrum for $22 \mathrm{BS}$ and ISL $=-93 \mathrm{dBm}$. This is because an increase in the number of active BSs and a decrease in the radiated signal level per BS is not enough to allow a high reuse of the spectrum due to the $23 \mathrm{~dB}$ stricter ISL constraint.

\subsection{White Space Availability}

Fig. 9 shows the white space distribution map (white space availability at each grid point in the area, see metric on Section 2.4.4) for the traditional Cognitive Radio network with noncoordinated spectrum management and 22 BS (Fig. 9a), the cloud-based centralized spectrum management with 22 BS (Fig. 9b) and the same network architecture with 33 BS (Fig. 9c).

The white space distribution maps in Fig. 9b and Fig. 9c correspond with the best results in terms of spectrum usage (denoted with marker 2 in Fig. 5 and 6b, respectively). The mean white space availability (accounting for the mean in the whole area) is $26.3 \%$ higher for the optimized proposed architecture than in the traditional Cognitive Radio network (Fig. 9b and 9a, respectively). Notice that in the city center the difference of white space availability can be higher. This is because the interference level is higher in the city center due to the BS locations distribution and the confluence of the radiation from more BSs. 

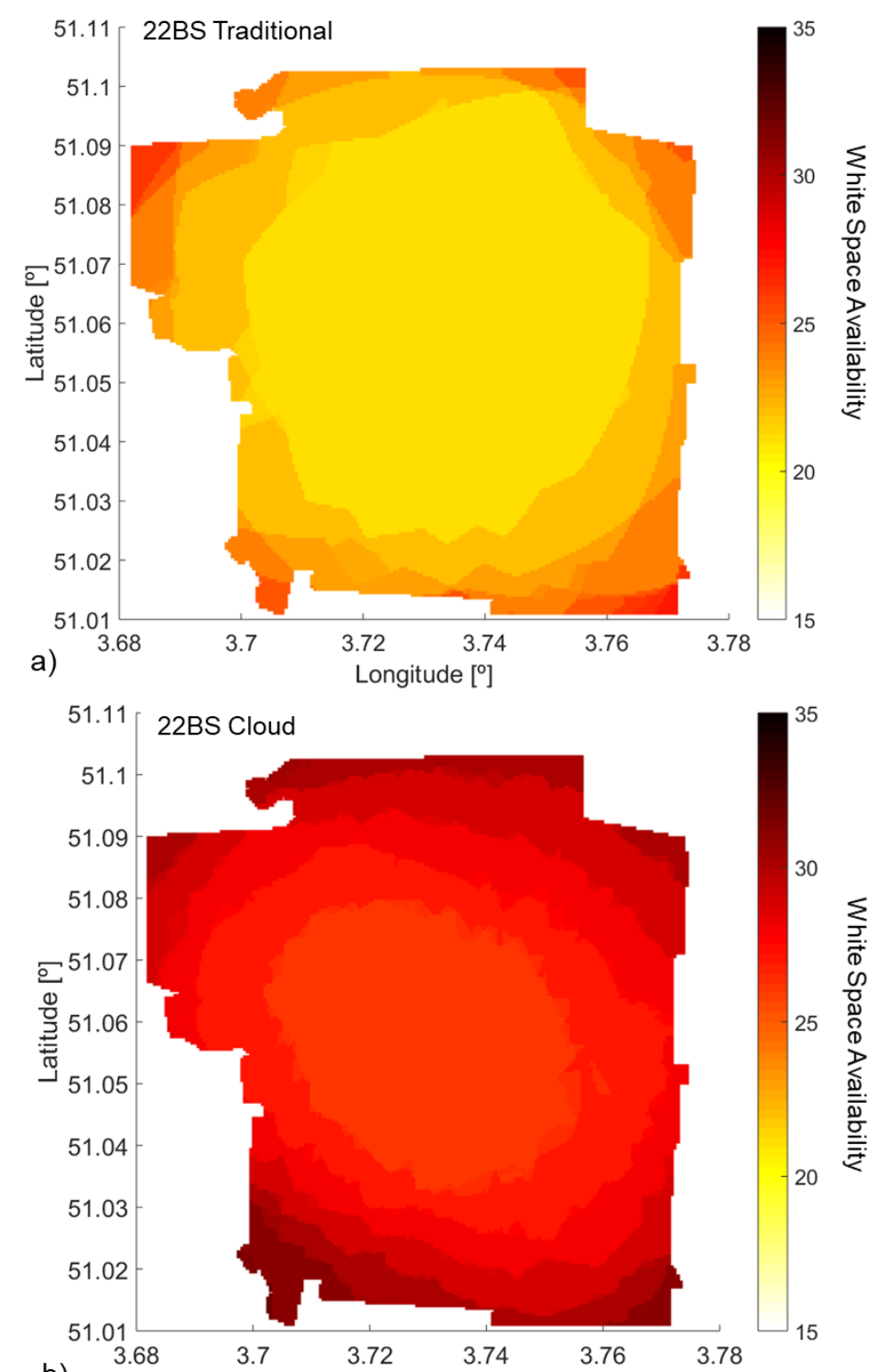

b)

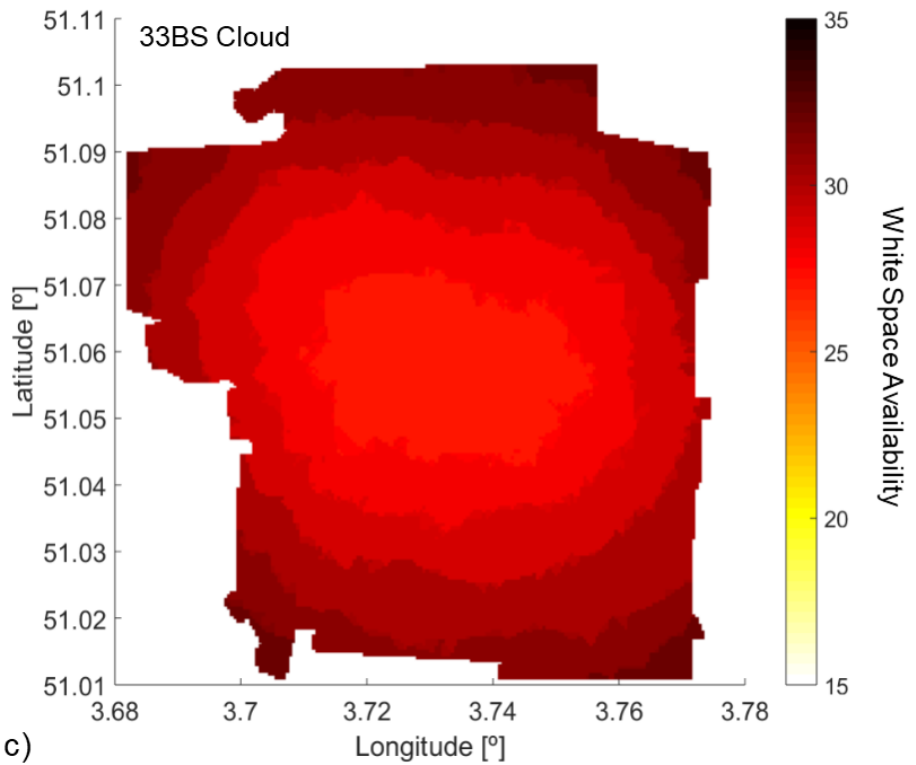

Fig. 9 White space availability distribution for a) the traditional Cognitive Radio architecture with 22 BSs, b) the proposed cloud architecture with 22 BSs and c) 33 BSs. 
Although the mean white space availability does not increase significantly with the number of BS infrastructure availability (approximately 2\% for a 50\% increase in BS availability), the gradient (speed) of white space availability is higher for 33 BS than for 22 BS (Fig. 9b and $9 \mathrm{c}$, respectively). This means more channels will be available in a region closer to Ghent if 33 BS locations are used for the green field network planning instead of 22 BS. This is because, although there are more radiating sources (active BSs), the radiation level per BS is lower. Hence the radiation is concentrated in a smaller area due to the environment path loss.

\section{Conclusions}

By means of a novel multi-objective optimization algorithm for Cognitive Radio networks, we quantified the advantages of cloud-based network management for Cognitive Radio technologies in comparison with a traditional distributed architecture. A Pareto efficiency modeling is performed for quantifying the trade-off among three KPIs: Power Consumption, Spectrum usage, and Exposure.

Compared to a traditional Cognitive Radio network, our proposed architecture and optimization algorithm reduces the network power consumption by $27.5 \%$, the average global exposure by $34.3 \%$, and spectrum usage by $34.5 \%$ for the best balance among the three KPIs. Even for the worst optimization case (i.e., worst achieved result of a single KPI for a certain Pareto coefficient), our solution performs better than the traditional architecture by $4.8 \%$ in terms of network power consumption, $7.3 \%$ in terms of spectrum usage, and $4.3 \%$ in terms of global exposure. For instance, when the power consumption has a weight of 0 in the fitness function, still the algorithm in the cloud-based architecture performs better than the traditional distributed architecture. This is because a better allocation of spectrum in the proposed architecture indirectly contributes to reducing the radiated power and power consumption.

For the cloud-based architecture, a higher BS infrastructure density (beyond the minimum that guarantees the intended spatial and temporal coverage) improves spectrum usage up to $5.6 \%$ and global exposure up to $16.3 \%$ but with a drawback in terms of network power consumption from $13.3 \%$ to $20.6 \%$.

Future research will consist of the experimental characterization of the data rate as a function of the interference for a dynamic interference constraint assessment for Cognitive Radio networks.

\section{Conflicts of Interest}

The authors declare that there is no conflict of interest regarding the publication of this paper.

\section{Funding Statement}

R. Martinez Alonso is supported by LACETEL and a doctoral grant from the Special Research Fund (BOF) of Ghent University, Belgium. 


\section{References}

[1] Shared Spectrum Company, "General Survey of Radio Frequency Bands - $30 \mathrm{MHz}$ to $3 \mathrm{GHz}$," Vienna, Virginia, 2010.

[2] V. Valenta, R. Marsalek, G. Baudoin and M. Villegas, "Survey on Spectrum Utilization in Europe: Measurements, Analyses and Observations," 5th International ICST Conference on Cognitive Radio Oriented Wireless Networks and Communications, 2010.

[3] J. van de Beek, J. Riihijarvi and A. Achtzehn, "TV White Space in Europe," IEEE TRANSACTIONS ON MOBILE COMPUTING, vol. 11, no. 2, pp. 178-188, 2011.

[4] A. Arteaga and A. Navarro, "Availability of TV White Spaces Using Spectrum Occupancy Information and Coverage Maps,” IEEE LATIN AMERICA TRANSACTIONS, vol. 14, no. 6, pp. 2588-2591, 2016.

[5] K. Kumar, A. Prakash and R. Tripathi, "Spectrum handoff in cognitive radio networks: A classification and comprehensive survey," Journal of Network and Computer Applications, vol. 61, no. February, pp. 161$188,2016$.

[6] Y. Saleem and M. Rehmani, "Primary radio user activity models for cognitive radio networks: A survey," Journal of Network and Computer Applications, vol. 43, no. August, pp. 1-16, 2014.

[7] M. Fadda, V. Popescu, M. Murroni, P. Angueira and J. Morgade, "On the Feasibility of Unlicensed Communications in the TV White Space: Field Measurements in the UHF Band," International Journal of Digital Multimedia Broadcasting, 2015.

[8] IEEE Computer Society, "IEEE 802.11af. Part 11: Wireless LAN Medium Access Control (MAC) and Physical Layer (PHY) Specifications. Amendment 5: Television White Spaces (TVWS) Operation.," IEEE, New York, US, 2013.

[9] IEEE Computer Society, "IEEE 802.22. Part 22: Cognitive Wireless RAN Medium Access Control (MAC) and Physical Layer (PHY) specifications: Policies and procedures for operation,” IEEE, New York, 2011.

[10] IEEE Computer Society, "IEEE 802.22b. Part 22: Cognitive Wireless RAN Medium Access Control (MAC) and Physical Layer (PHY) Specifications: Policies and Procedures for Operation in the TV Bands," IEEE, New York, 2015.

[11] ETSI, "TR 103067 Reconfigurable Radio Systems (RRS); Feasibility study on Radio Frequency (RF) performance for Cognitive Radio Systems operating in UHF TV band White Spaces," ETSI, France, 2013.

[12] L. Montsi, L. Mfupe, F. Mekuria and M. D., "A real-time big-data system for smart spectrum sharing: Enabling affordable broadband in 5G for underserved communities," in Global Wireless Summit, Cape Town, South Africa, 2014.

[13] G. L. Stuber, S. M. Almalfouh and D. Sale, "Interference Analysis of TV-Band Whitespace," Proceedings of the IEEE, vol. 19, no. 4, pp. 741-754, 2009.

[14] V. Popescu, M. Fadda, M. Murroni, J. Morgade and P. Angueira, "Co-Channel and Adjacent Channel Interference and Protection Issues for DVB-T2 and IEEE 802.22 WRAN Operation," IEEE TRANSACTIONS ON BROADCASTING, vol. 60, no. 4, pp. 693-700, 2014.

[15] S. J. Shellhammer, "Spectrum sensing in IEEE 802.22," in IAPR Work on Cognitive Inf. Process, San Diego, 2008.

[16] C. You, H. Kwon and J. Heo, "Cooperative TV spectrum sensing in cognitive radio for Wi-Fi networks," IEEE Transactions on Consumer Electronics, vol. 57, no. 1, pp. 62-67, 2011.

[17] W. Prawatmuang, D. K. C. So and E. Alsusa, "Sequential Cooperative Spectrum Sensing Technique in Time Varying Channel," IEEE TRANSACTIONS ON WIRELESS COMMUNICATIONS, vol. 13, no. 6, pp. 3394-3405, 2014.

[18] N. Tadayon and S. Aissa, "A Multi-Channel Spectrum Sensing Fusion Mechanism for Cognitive Radio Networks: Design and Application to IEEE 802.22 WRANs," IEEE TRANSACTIONS ON COGNITIVE COMMUNICATIONS AND NETWORKING, 2016.

[19] A. Ebian, B. Abdelhamid and S. El-Ramly, "New measurement matrix for compressive sensing in cognitive radio networks," IET Communications, vol. 12, no. 11, pp. 1297-1306, 2018.

[20] T. Jiang, D. Grace and P. Mitchell, "Efficient exploration in reinforcement learning-based cognitive radio spectrum sharing," IET Communications, vol. 5, no. 10, p. 1309-1317, 2011.

[21] S. K. Gottapu, N. Kapileswar, P. V. Santhi and V. K. R. Chenchela, "Maximizing Cognitive Radio Networks Throughput using Limited Historical Behavior of Primary Users," IEEE Access, vol. 6, pp. 12252 - 12259, 2017. 
[22] V. Popescu, M. Nitti, D. Calaver, M. Razvan Ardelean and M. Fadda, "QoS Evaluation for Indoor Broadcasting Services using an IoT platform," in BMSB, Valencia, Spain, 2018.

[23] M. Deruyck, E. Tanghe, D. Plets, L. Martens and W. Joseph, "Optimizing LTE wireless access networks towards power consumption and electromagnetic exposure of human beings," Computer Networks, no. 94, pp. 29-40, 2016.

[24] M. Deruyck, W. Joseph, E. Tanghe, D. Plets and L. Martens, "Designing Green Wireless Access Networks: Optimizing towards Power Consumption versus Exposure of Human Beings," Proceedings of the 11th International Symposium on Wireless Communications Systems, pp. 224-228, 2014.

[25] D. Plets, W. Joseph, K. Vanhecke and L. Martens, "Exposure optimization in indoor wireless networks by heuristic network planning," Progress In Electromagnetics Research, vol. 139, pp. 445-478, 2013.

[26] J. Wu, "Green wireless communications: from concept to reality," IEEE Wireless Communications, vol. 19, no. 4, pp. 4-5, 2012.

[27] J. Wu, S. Rangan and H. Zhang, Green Communications: Theoretical Fundamentals, Algorithms, and Applications, Florida, USA: CRC Press, 2016.

[28] M. Zheng, L. Chen, W. Liang, H. Yu and J. Wu, "Energy-efficiency Maximization for Cooperative Spectrum Sensing in Cognitive Sensor Networks," IEEE Transactions on Green Communications and Networking, vol. 1, no. 1, pp. 29-39, 2017.

[29] M. T. Mehari, E. De Poorter, I. Couckuyt, D. Deschrijver, G. Vermeeren, D. Plets, W. Joseph, L. Martens, T. Dhaene and I. Moerman, "Efficient Identification of a Multi-Objective Pareto Front on a Wireless Experimentation Facility," IEEE Transactions on Wireless Communications, vol. 15, no. 10, pp. 6662 $6675,2016$.

[30] M. Katz, F. H.P. Fitzek, D. E. Lucani and P. Seeling, "Mobile Clouds as the Building Blocks of Shareconomy: Sharing Resources Locally and Widely," IEEE Vehicular Technology Magazine, vol. 9, no. 3, pp. 63-71, 2014.

[31] N. Barr, Economics of the Welfare State, United Kindom: Oxford University Press, 2012.

[32] R. Martinez Alonso, D. Plets, M. Deruyck, G. Guillen Nieto, L. Martens and W. Joseph, "TV White Space and LTE Network Optimization towards Energy Efficiency in Suburban and Rural Scenarios," IEEE Transactions on Broadcasting, 2017.

[33] V. Nadege, D. Plets, Y. Corre, G. Vermeeren, W. Joseph, L. M. S. Aerts and J. Wiart, "A novel method to assess human population exposure induced by a wireless cellular network," Bioelectromagnetics, vol. 36, no. 6, p. 451-463, 2015.

[34] SATBROADCAST, "Digital terrestrial transmitters and muxes in Belgium," SATBROADCAST, 2019.

[35] TV Radio Nord, “Reception Hertzianne dans le Nord Pas de Calais,” TV Radio Nord, 2018. [Online]. Available: http://www.tvradio-nord.com/emetteurs/emetteurs_belge/carte_begique.html. [Accessed July 2018].

[36] ITU-R, "Recommendation ITU-R P.1546-5: Method for point-to-area predictions for terrestrial services in the frequency range $30 \mathrm{MHz}$ to $3000 \mathrm{MHz}$," ITU, Geneva, 2013.

[37] D. Plets, W. Joseph, E. Tanghe, L. Verloock and L. Martens, “Analysis of propagation of actual DVB-H signal in a suburban environment," IEEE Antennas-and-Propagation-Society International Symposium, Vols. 1-12, pp. 1839-1842, 2007. 